\title{
Hybrid-Image Visualization for Large Viewing Environments
}

\author{
Petra Isenberg, Pierre Dragicevic, Wesley Willett, Anastasia Bezerianos, Jean-Daniel Fekete
}

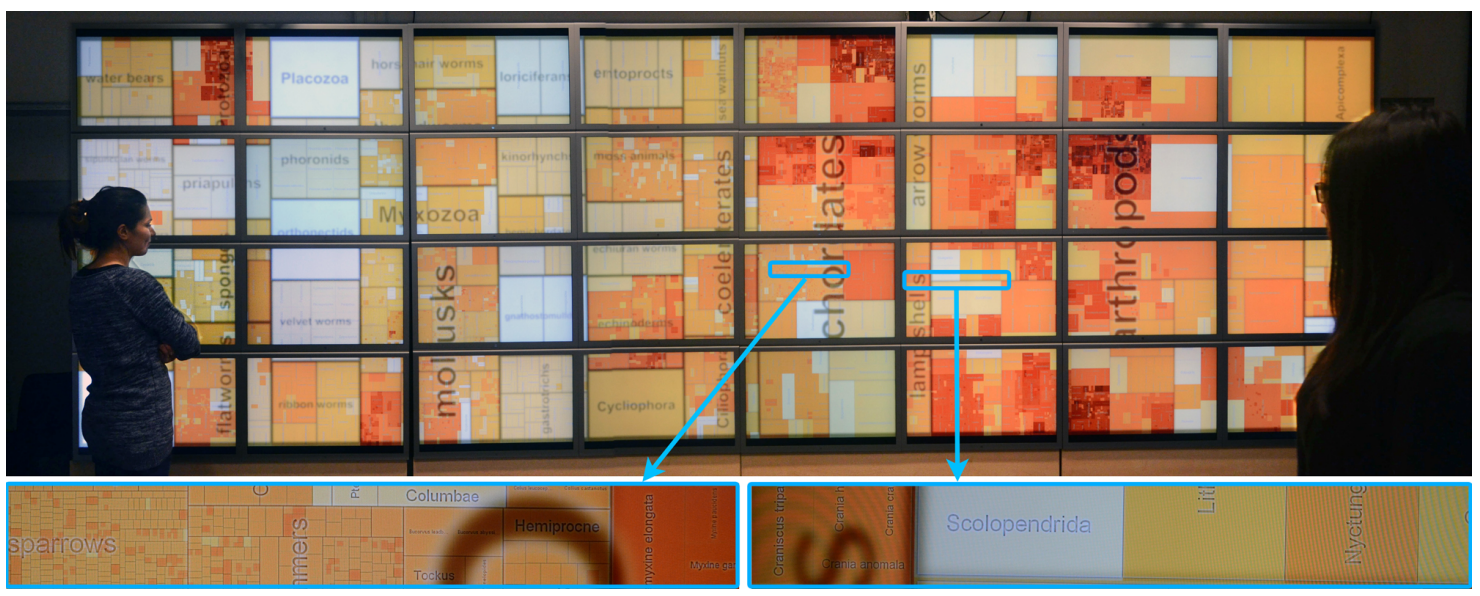

Fig. 1. Two viewers looking at a hybrid-image treemap and two close-up views from the center of the display.

\begin{abstract}
We present a first investigation into hybrid-image visualization for data analysis in large-scale viewing environments. Hybrid-image visualizations blend two different visual representations into a single static view, such that each representation can be perceived at a different viewing distance. Our work is motivated by data analysis scenarios that incorporate one or more displays with sufficiently large size and resolution to be comfortably viewed by different people from various distances. Hybrid-image visualizations can be used, in particular, to enhance overview tasks from a distance and detail-in-context tasks when standing close to the display. By using a perception-based blending approach, hybrid-image visualizations make two full-screen visualizations accessible without tracking viewers in front of a display. We contribute a design space, discuss the perceptual rationale for our work, provide examples, and introduce a set of techniques and tools to aid the design of hybrid-image visualizations.
\end{abstract}

Index Terms-Multi-scale, large displays, hybrid images, collaboration, visualization

\section{INTRODUCTION}

We explore the use of hybrid-image visualization for data analysis in large-scale viewing environments. These environments incorporate a display of large size and resolution (wall display, billboard, or large poster) that can be comfortably viewed from different distances. In such an environment, a hybrid-image visualization can show two representations such that-in the same view and without tracking viewers in the space-viewers close to the display seen detailed information while viewers at a distance instead see a global overview. Hybridimage visualizations are based on a technique called hybrid images [30] that blends two images after applying frequency filters to achieve distance-dependent perception.

Our work is primarily motivated by collaborative viewing situations with several viewers situated in front of high-resolution wall-sized displays. Wall-sized displays (e.g. [8]) offer several benefits for data analysis: their large viewing space and high pixel count allow for the simultaneous view, comparison, and exploration of large amounts of data in one shared view, while being well suited to collaborative data analysis due to the large physical viewing space that can be comfort-

- Petra Isenberg, Pierre Dragicevic, Wesley Willett, and Jean-Daniel Fekete are with INRIA, E-mail:(petra.isenberg | pierre.dragicevic | wesley.willett | jean-daniel.fekete)@inria.fr.

- Anastasia Bezerianos is with LRI (Univ. Paris-Sud \& CNRS) \& INRIA E-mail: anastasia.bezerianos@lri.fr.

Manuscript received 31 March 2013; accepted 1 August 2013; posted online 13 October 2013; mailed on 5 October 2013.

For information on obtaining reprints of this article, please send

e-mail to:tvcg@computer.org. ably shared. In particular, our hybrid-image visualization techniques are motivated by findings on the importance of physical navigation in front of wall displays [2]. Physical navigation allows viewers to pan and zoom through locomotion. During a physical pan a viewer can inspect a new subset of the data by re-orienting his or her viewpoint. During physical zoom, a viewer steps closer to or farther away from a display while keeping the viewpoint steady. This zoom changes the perception of the visual variables encoding the data. Imagine a viewer stepping back from a close viewing distance to a far one while keeping his/her eyes fixed at the same position on the display. This physical zoom-out can have several effects on the perception of the data encodings: (a) some parts of the data will begin to form visible clusters and will be perceived as a unit ([19, Chapter 7]), (b) some parts of the data previously visible will be lost as they reach the limits of visual acuity or contrast sensitivity [40], and (c) the perception of color [36] and quantitative estimation of magnitude of visual variables can change [10]. Given these changes in perception, it is clear that the effectiveness of visualizations viewed from afar hinges on the visual aggregation of particular data encodings and whether the data analysis task can benefit from visually aggregated data.

Several techniques are available for designing view-distancedependent (henceforth: distant-dependent) information visualizations. Our design space in Section 3 details different options. We focus on hybrid images as a still underexplored technique for information visualization. We show how hybrid images can be used to overlay visualizations designed for near and far viewing distances such that, from close to the display, the encodings for far viewing distances practically disappear. Meanwhile, the information meant for close inspection does not hinder the overview from afar. Hybrid images have the advantage that the whole display space can be used for drawing both local and 
global information. They also do not require interactivity to work and thus are different from techniques that require the tracking of viewers in a space. Our paper makes four main contributions:

- A design space of view-distance dependent encodings,

- A detailed discussion of the perceptual background and rationale for using hybrid images for creating distance-dependent views,

- Example visualizations and a summary of encoding techniques,

- A set of tools to aid the design of hybrid-image visualizations.

\section{Related Work}

Hybrid-image visualizations, as a distance-dependent visual encoding, belong to a family of images that can be seen in more than one way. We review this family of images and its related work in Section 3. Here we focus on related research on distance-dependent viewing and interaction most closely related to our main motivation.

The idea to encode information differently based on a viewer's physical position has been used in recent work $[21,25]$ on color perception properties of LCD screens. Using specific renderings the authors created images such that multiple viewers sitting at different locations around the screen could see different information. Although relevant to our motivation, this work takes advantage of specific hardware characteristics and is thus difficult to generalize. Zoomable interfaces [9] or in general multi information resolution interfaces [26] are another approach related to distance-dependent viewing. While with zooming a viewer's virtual rather than the physical viewpoint changes, techniques such as semantic zooming still display different information or representations of the data depending on the virtual distance (zoom level). In contrast to our motivation, however, zoom-based techniques are not well suited for collaborative scenarios as they typically change the whole view. Nevertheless the idea to encode different information for different viewing distances and the idea to display different resolutions of data side-by-side [26] has inspired several of our examples in Section 5.1. Similarly, recent work applying proxemics to the design of interfaces has been influenced by the idea of semantic zoom. Greenberg et al. [20] describe a set of five proxemic dimensions (distance, orientation, movement, identity, location) that help to measure proxemics - ways in which people mediate their interactions with other people through interpersonal distance-when applied to ubiquitous computing environments. Distance is the dimension most related to our work. Distance between humans and devices can be measured continuously or discretely through zones. The idea of zoning has been previously used in HCI to render content on a display depending on viewers' physical location. In Vogel and Balakrishnan's [39] ambient display, content is rendered depending on which of a set of four zones a viewer is located in. In an application for a video player Ballendat et al. [3] combine both continuous and discrete distance measures to display a different media player interface to a viewer. This work adapts content interactively based on tracking viewers in contrast to ours that encodes information in one view for multiple viewing distances.

In information visualization, the role of distance of humans to a display has been explored in relation to perception. Yost and North [45] found their visualizations to scale well for both detailed and overview finding tasks on wall displays, and noted that spatial encoding of information was particularly important for performance. They hypothesized that the resulting visual aggregation led to reduced need for physical navigation, which improved performance. In a follow-up experiment Yost et al. [44] studied how scaling visualizations beyond visual acuity affected user performance. For almost all tested tasks they found performance improvements and argue for design guidelines that take visual aggregation and physical navigation into account. More recently, Endert et al. [16] explored how a viewer's distance from a large display influences the visual aggregation of displayed information, and found encodings based on a color ramp to visually aggregate particularly well across viewing distances for a visual search task. Finally, Bezerianos and Isenberg [10] examined how basic visual encodings are perceived under different viewing distances and angles and found large discrepancies in a simple comparison task when viewers were positioned close to a large wall display. They observed that comparison errors tended to decrease if appropriate physical navigation was used.

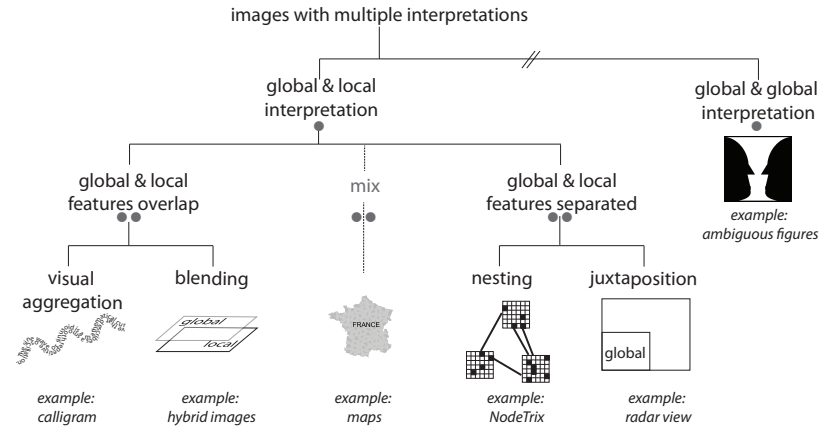

Fig. 2. Design space of images that can be seen in more than one way. The left branch includes viewing-distance dependent encodings. Bullet points refer to hierarchy levels in analogy to Section 3.

This body of work stresses the importance of physical movement in front of wall-sized displays and how different viewing distances are appropriate for different tasks. As this past work mainly studied the effect of viewing distance and visual aggregation, it is orthogonal to the work presented here that attempts to explicitly take advantage of different viewing distances to encode additional information.

\section{IMAGES THAT CAN BE SEEN IN MORE THAN ONE WAY: A DESIGN SPACE}

Hybrid-image visualizations are related to other images that can be seen in more than one way. There is a large body of work in this area, and many types of information visualizations and many art pieces are susceptible to different perceptual interpretation. Fig. 2 gives an overview of our design space. In the following subsections bullet points encode the hierarchy level as shown in the figure:

- Several global interpretations: On the highest-level we distinguish images based on the type of perceptual interpretation they support. A large set of optical illusions fall into the category of images that have several global interpretations. That is, the whole image can be viewed as showing one object or another. Ambiguous figures are a typical example. The Rubin's reversible face-vase shown in Fig. 2 is one famous example. These images have long been known in perceptual psychology and are used to study perceptual reversal (the alternation between two mutually exclusive perceptual states) [15]. Gestalt psychologists studied these images to understand how humans distinguish between figure and ground [19]. Reversible or ambiguous figures do not seem to favour interpretations based on viewing distance which distinguishes them from the remainder of images in our design space. - Local and a global interpretations: The group that includes hybrid images offers both a local and a global interpretation. That is, information at a fine spatial scale (local information) is available when viewing an image up-close and coarse-scale (global) information is available when viewing it from afar. Thus, images in this category offer the possibility to encode information for distance-dependent viewing. We focus here on single-view techniques and specifically exclude several others that are related such as: (a) interactive techniques, e.g. (semantic) zoom, (b) multi-display environments with separate displays for local and global information, and (c) techniques that have no information-carrying units on a fine spatial scale. For example, ASCII art, maze images [32], Artistic Screening [31], and other drawing techniques (e.g., hatching or half-toning) make up larger images from many small visual components that are only visible up-close. Close-up inspection, however, does not reveal additional information and, thus we exclude these images and techniques in this design space. - Global and local features overlap: How global and local features are mixed on the display is the distinguishing factor in the category of images with both a local and global interpretation.

The first group of techniques takes advantage of perceptual aggregation-local features forming global patterns when seen from afar. These global patterns appear naturally based on properties of visual perception such as the laws of grouping from Gestalt theory [19], visual acuity, or contrast sensitivity. For example, in a scatterplot, individual dots - given a correctly chosen background contrast and size- 
will begin to form clusters naturally at a distance. In information visualization several projects have made use of such natural aggregations. Wong et al. [42], for example, encode extra information on node-link diagrams by using specifically laid out text labels to represent both the links and the nodes of the graph. Afzal et al. [1] presented automatic typographic maps where typography forms certain map features. Nacenta et al.'s FatFonts [28] can be used to fill regions with numbers that represent the data while the typeface of the numbers is such that their area (amount of 'ink') is proportional to the represented number. Natural groupings and aggregations have also been extensively used in art. Examples are the paintings "All is Vanity" by C. Allan Gilbert or the famous Arcimboldo paintings of heads made up of vegetables or fruits. Several of these artistic dual perception images "favour" one type of interpretation depending on viewing distance-for example in Gilbert's work the woman is more prominent when viewed up close, while the scull appears more strongly when viewed from further away. Photomosaics [6] are another artistic example of images obtained by putting together small colored fragments that are themselves images (tiles). From afar the tiles blend visually to create a coherent imagewhen seen close together the images on each individual tile form the local information. Related to photomosaics are Word Pictures where the stylized text of tag clouds is arranged to form images, as well as traditional Calligrams or Micrography - the use of stylized text arranged in a way that creates a visual image usually related to the text. One disadvantage of visual aggregation-based techniques is that the encodings are hard to control. For example, while points in a scatterplot may form large clusters, viewing outliers can become impossible from afar limiting the effectiveness of the encoding for this type of overview task. Furthermore, as several researchers have pointed out $[16,36]$, we need more research on how features visually aggregate.

The second group of techniques in this category overlap visual features by blending both global and local information. A simple approach is alpha-blending. Maps sometimes use this technique to place large labels for distant viewing on top of the local topology layer. Extensions of alpha-blending have been proposed to selectively blend color components and features from two images in order to improve legibility, although not for multiple viewing distances [7, 23, 27]. Hybrid images - which we discuss in detail in Section 4-are an example where local and global information is frequency-filtered and then blended in order to ensure distance-dependent viewing.

- Global and local features separated: Images in this category have specifically assigned regions for fine and coarse-scale information. One set of techniques nests local information inside features that form the global view. NodeTrix [22], for example, encodes small matrix visualizations inside the nodes of a large network graph. Given the right rendering size and color choices, the larger network graph forms the global view while the local connectivity encoded inside the nodes forms local information available for close inspection. A second group of techniques uses juxtaposition to place both local and global information. A large number of focus-(and/in)-context techniques fall into this category. Radar views, for example, provide small windows showing a global view next to a viewing area with local information. Other techniques interweave information even more strongly. Imagine a bulletin board or poster layout with large title and large slogans to draw attention from afar and detailed information in the form of text boxes and smaller images for close inspection. The amount of interweaving may make a practical difference when it comes to the property of distancedependence: when spaces for local and global information are clearly separated, one viewer could stand close to the detailed display and another could stand further to get a global view. When local and global spaces are interwoven across the display, navigating between pieces of local information may be practically much more difficult. A general disadvantage of all techniques in this category is that parts of the display are reserved for either close or far viewing distances reducing the display space for either type of information.

- Mixed: Several examples use more than one of the techniques described above. In maps, for example, countries are often color-coded and blended with local information while global and local labels are juxtaposed across the map. Pixel Bar Charts [24] are another mixed encoding that can be made distance-dependent. It renders individual data items as pixels nested inside the bars and colors the pixels according to data properties. When pixels are sorted according to color, they visually aggregate from afar into regions that give overview or global information. An example from HCI is the ambient display by Vogel and Balakrishnan [39]. Here data to be viewed from afar is alphablended with local information but local information is also restricted just to the lower portion of a display to afford easy interaction.

In summary, with this design space we have shown that there is a large number of techniques available for creating distance-dependent information visualizations. Many of these have already been explored, although not always under the umbrella of distance-dependent encoding. We chose to focus on hybrid images as one under-explored technique for information visualization.

\section{Rationale For Hybrid-Image Visualizations}

In this section, we present background information on hybrid images and motivate their use in the context of information visualization in large viewing environments.

\subsection{Spatial Frequency Analysis}

Key to the creation of hybrid images is the notion that any image can be represented as a 2D-array of color values (the spatial domain) and also as a weighted sum of sines and cosines of different frequencies (the frequency domain) [11]. With a Fourier transform, we can convert images from the spatial domain into a 2D frequency domain. Computing the rotational average of the $2 \mathrm{D}$ frequency representation produces a $1 \mathrm{D}$ frequency series, which can be plotted as a power spectrum.

To express spatial frequencies we use two units: the unit pixels per cycle (ppc) is the spatial period expressed in pixels. For example, a series of gradually-varying black and white stripes (a sinusoidal grating) where each stripe is 4 pixel wide corresponds to a period of $8 \mathrm{ppc}$. We also use the standard unit of cycles per degree (cpd) to capture what the observer sees. This corresponds to the number of periods per degree of visual angle. For a display of pixel size $p$ and a viewer at distance $d$ facing the image, ppc can be converted into cpd using the following formula: $c p d=\pi /\left(360 \cdot \tan ^{-1}\left(\frac{p}{2 d} \cdot p p c\right)\right)$.

Frequency-domain representations can be used to measure the frequencies present in an image and determine when they are likely to be visible. Moreover, certain image filters can be described as operations in the frequency domain. For example, blurring an image amounts to applying a low-pass filter, i.e., attenuating its high-frequency components while leaving its low-frequency components mostly intact. A high-pass filter is the opposite operation, while a band-pass filter attenuates frequencies both above and below a certain frequency range.

\subsection{Origins of Hybrid Images}

A body of literature in psychology suggests that the human perceptual system analyzes images at multiple scales through a collection of bandpass filters, each narrowly tuned to a specific frequency band [12, 29]. As part of this body of work, Oliva and Schyns introduced hybrid stimuli as an experimental tool for studying multi-scale perception processes during scene recognition [34]. Hybrid stimuli were created by blending a low-pass filtered image with a high-pass filtered image. Using recognition tasks on hybrid stimuli, they showed that visual information is first extracted from large-scale features then refined with small-scale features, but that humans can also selectively attend to specific frequency ranges depending on the task [29, 34, 35].

In these studies hybrid stimuli were only presented from a fixed distance and for brief periods of time. Oliva and colleagues later coined the term hybrid image in a short computer graphics paper, where they discuss the use of hybrid stimuli as images that can be perceived differently depending on viewing distance [30]. They provide basic psychological grounding, briefly discuss how to design effective hybrid images and mention a few applications. Since their introduction, hybrid images have been used in other contexts, showing differently morphed faces on ambient displays for the elderly [43] or to improve image morphing [17]. We know of no information visualization applications. 


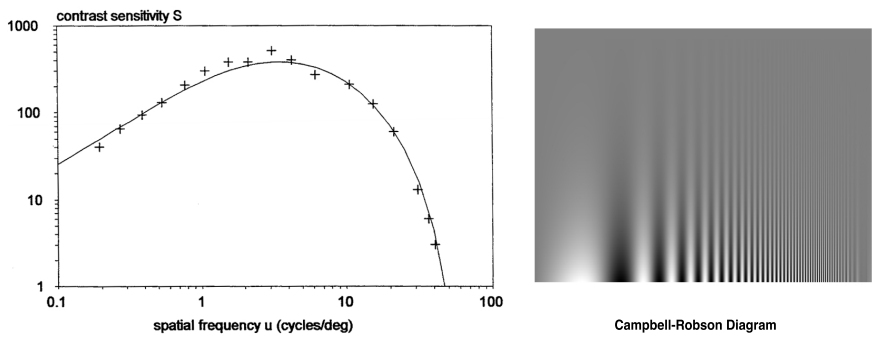

Fig. 3. Left: a typical contrast sensitivity function (CSF), after [4]. Right: Sinusoidal gratings varying in frequency and contrast.

Although Oliva et al.'s [30] article is our main source of inspiration, it hardly provides enough details to help designers create effective hybrid-image visualizations. The article mostly focuses on the entertaining potential of hybrid images and of the possible use cases mentioned, only one (text obfuscation) captures an actual application. Design considerations are very general and mostly focus on how to have the two percepts entirely exclude each other.

\subsection{Visual Perception in Large Viewing Environments}

Next, we provide basic theoretical grounding for the use of hybridimage visualizations. We explain how viewers perceive visual content in large viewing environments depending on their location. Since we are only interested in the effect of distance we assume viewers facing the display and we ignore issues related to perspective distortion [10].

\subsubsection{Feature Visibility}

The visibility of a particular object depends on many factors; one among these is the observer's visual acuity. Visual acuity can be assessed by vision tests like Snellen Charts, which measure the visual angle below which objects such as letters become invisible or illegible [38]. These models do not capture essential features of human vision, such as the fact that low-contrast objects are harder to perceive than high-contrast objects, or that very large-scale features are perceived less strongly than medium-scale features (lateral inhibition [4]).

A widely-accepted model of visual sensitivity is the contrast sensitivity function (CSF). It models the response of the human visual system with spatial frequency and the contrast of visual stimuli [4]. Fig. 3-left shows the shape of a typical CSF, where the $\mathrm{x}$-axis is the frequency and the $y$-axis is the visual sensitivity for that frequency. The higher the sensitivity the higher the visual response generated by the stimulus, or equivalently, the less contrasted a stimulus needs to be to be seen. Although the exact shape of the CSF depends also on environmental factors, humans generally exhibit a peak of sensitivity at 3-4 cpd. Sensitivity rapidly decreases as spatial frequency increases, and stimuli become invisible at about $60 \mathrm{cpd}$ due to limits in visual acuity. Below 3-4 cpd, sensitivity decreases due to lateral inhibition. The types of stimuli captured by the CSF are illustrated by the sinusoidal gratings in Fig. 3 right, also known as Campbell-Robson Diagram.

Fig. 4 puts CSFs in context by showing the visibility of small- and large-scale features as a function of viewer distance. We applied Barten's [4] computational model of CSF for an average subject ${ }^{1}$ to the WILD display. ${ }^{2}$ [8]. The $\mathrm{x}$-axis on Fig. 4 is the viewer's distance to the display and the $y$-axis is the visibility of the feature (corresponding to the output of the CSF function shown on a linear scale). Features are high-contrast sinusoidal gratings whose size varies from 2 ppc (corresponding to single-pixel black and white stripes) to $256 \mathrm{ppc}$. It can be seen that gratings of $2 \mathrm{ppc}$, i. e., the smallest displayable feature, are visible from close by but rapidly become hard to see and finally disappear around $1.5 \mathrm{~m}$. Features of $4 \mathrm{ppc}$ disappear at about $3 \mathrm{~m}$. In contrast, large features of 128 or $256 \mathrm{ppc}$ are more prominent from far and

\footnotetext{
${ }^{1}$ We used Barten's default constants and $p=1.240 \times 10^{6}, X_{0}=30 \mathrm{deg}$ and $L=100 \mathrm{~cd} / \mathrm{m}^{2}$.

${ }^{2}$ The WILD wall (Fig. 1) has $8 \times 430$ " Apple Cinema displays of $2560 \times 1600 \mathrm{px}$ each (100ppi). It has a maximum viewing distance of $4 \mathrm{~m}$.
}

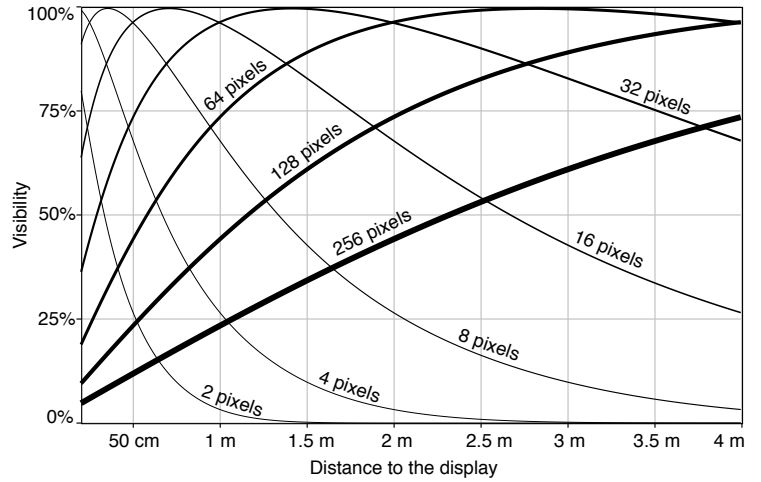

Fig. 4. Visibility of high-contrast gratings on the WILD display as a function of grating size $(2-256 \mathrm{ppc})$ and viewer distance $(20 \mathrm{~cm}-4 \mathrm{~m})$.

the closer the viewer gets, the fainter they become. Finally, mediumsized features of 8-64 ppc have individual "ideal" viewing distances. Fig. 4 clearly shows that different image features are salient at different distances, and that the pattern is more complex than the common notion that small details disappear from far. When moving away from the ideal viewing distance, feature visibility actually decreases more rapidly when moving towards the display than away from the display.

\subsubsection{Feature Information Capacity}

Fig. 4 suggests that large-scale features may need very large distances to be seen under ideal conditions (e. g., about $4 \mathrm{~m}$ for $128 \mathrm{ppc}$ ). These features can be easily displayed on a single computer screen and yet, users of desktop computers rarely feel compelled to step back $4 \mathrm{~m}$ to attend to large-scale features. ${ }^{3}$ Note however that within a given display area, much less information can be shown with large-scale features than with small-scale features. Consider a glyph-based visualization. If each glyph uses a frequency band of 2-32 ppc, doubling glyph size (yielding periods of 4-64 ppc) divides the number of displayable glyphs by 4 . More generally, a feature of period $p / k$ can convey visual content that is $2^{k}$ denser on the display than a feature of period $p$.

However, the area of a large display that can be effectively used to convey information also depends on viewing distance. Zooming on a regular display provides an analogy: when zooming in, more details can be seen but the same amount of visual information moves outside the viewport [18]. Although when moving in natural settings there is no clear viewport beyond which visual information gets abruptly "cropped", a similar phenomenon occurs.

The human field of view spans a wide angle, but only a tiny fraction can be used for inspecting details. Visual inspection therefore requires eye movements, as well as head movements when the visual angle subtented by the object is large enough. Head movements are uncomfortable and are usually avoided when reading text-visual angles of 20 to $40^{\circ}$ are considered comfortable for reading text on paper [37], and also correspond to typical viewing distances on computer screens [14]. It is reasonable to assume that viewers of large display environments will also try to have their region of interest span a visual angle of no more than $40^{\circ}$, and when this region becomes too wide, they will likely move away from the display in order to avoid repetitive head movements and possible perspective distortion [10].

When a large display covers more than $40^{\circ}$ of visual angle, the area of the display that can be used to show information is proportional to $d^{2}, d$ being the viewer's distance. When the whole display area is visible, moving further does not add more display surface.

For a given feature seen from a given distance, we define information capacity as the product of the feature's density on the display and the usable display area from this distance. Fig. 5 shows feature visibilities from Fig. 4 corrected for information capacity. ${ }^{4}$ The dashed enve-

\footnotetext{
${ }^{3}$ The largest grating a single Apple Cinema Display can show is $2560 \mathrm{ppc}$, for which the ideal viewing distance would be more than 100 meters!

${ }^{4}$ The CSF functions were multiplied by information capacity. We considered a viewer facing the display center with an effective viewing angle of $40^{\circ}$.
} 


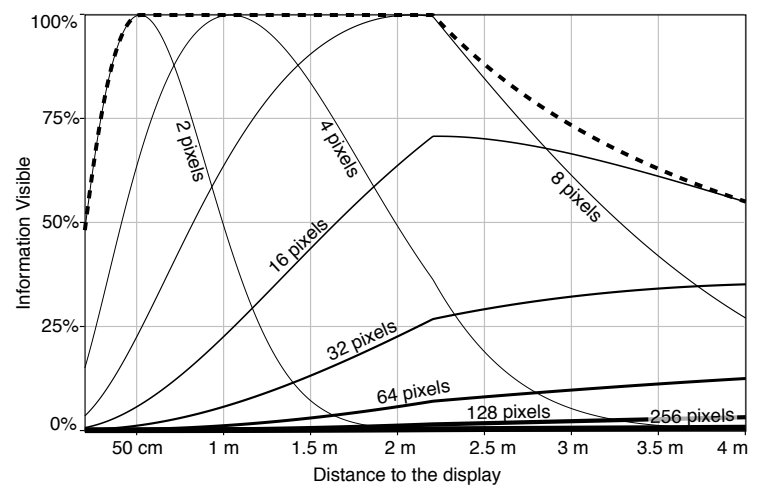

Fig. 5. Visibility of high-contrast gratings on the WILD display corrected for information capacity as a function of grating size and viewer distance.

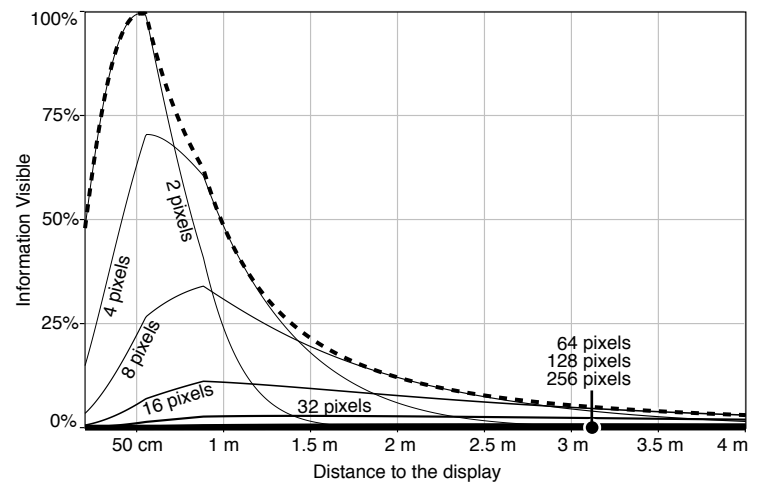

Fig. 6. Visibility of high-contrast gratings on a desktop display corrected for information capacity, as a function of grating size and distance.

lope gives the value of the feature yielding the best tradeoff between visibility and information capacity. The stable region between $60 \mathrm{~cm}$ and $2.2 \mathrm{~m}$ is due to the effects of feature density on the display and of usable display area cancelling out. Beyond $2.2 \mathrm{~m}$, moving further reduces the quantity of perceivable visual information due to the unused visual space above and below the display. ${ }^{5}$ Still, features larger than $32 \mathrm{ppc}$ become easier to see. At $60 \mathrm{~cm}$, getting closer does not provide clear benefits, since the viewable area keeps decreasing while the LCD display cannot show more details.

As a basis for comparison, Fig. 6 shows the same simulation on a 30" desktop display. Here, no stable region exists where different information can be conveyed at different distances. Instead, it exhibits a single peak for 2-pixel features at about $60 \mathrm{~cm}$ (a typical viewing distance in desktop settings [14]). For inspecting features of 8-16 pixels, standing at $80 \mathrm{~cm}$ seems preferrable since less head movements are required while the features are still clearly visible. Larger features are in principle easier to see from large distances (see Fig. 4) but the amount of information they can convey on a single display is too negligible to provide clear benefits. When standing closer than $60 \mathrm{~cm}$, features of 2-4 ppc may also be easier to see (see Fig. 4), but the display area that can be comfortably inspected also gets much smaller.

In summary, high-resolution large viewing environments lend themselves well to information visualization because they can convey different, complementary visual information at different distances. Not only can a large amount of visual information be displayed in terms of total amount of pixels, but viewers can also integrate and absorb this large quantity of information through locomotion. That being said, not all visualizations are equally effective at conveying information in these environments. Visualizations must be designed to convey legible, useful and possibly complementary information at different distances.

\subsubsection{Two Suboptimal Visualizations}

Visualization designers are used to setups involving fixed viewing distances and limited display resolution. Given the extremely large

${ }^{5}$ The curve decreases faster starting from $7 \mathrm{~m}$ (not shown in Fig. 5) due to the fact that the display's horizontal visual angle also falls below $40 \mathrm{deg}$.
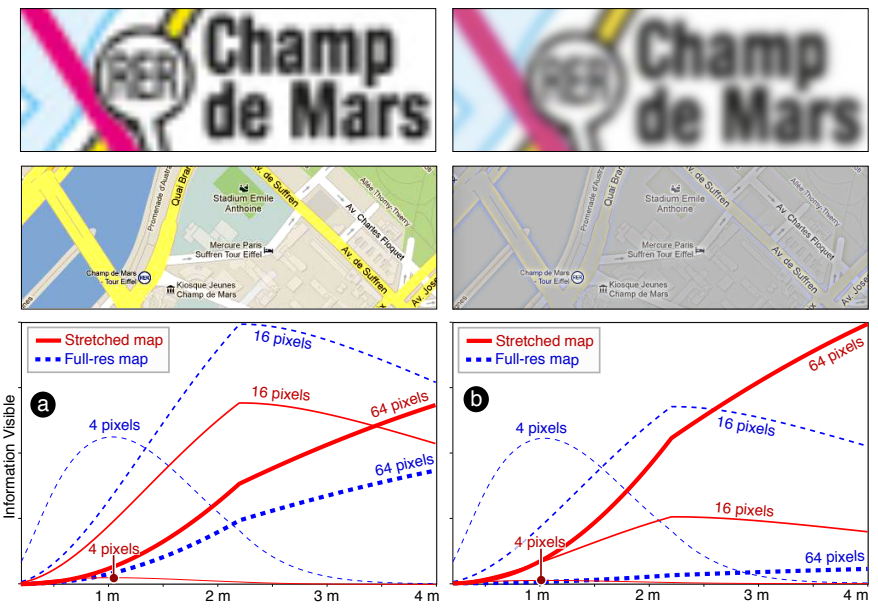

Fig. 7. A stretched subway map and full-resolution city map before filtering (left) and after (right). Charts show the frequency content of the stretched map (red) and a full-resolution map (blue) as a function of viewing distance both before filtering (a) and after (b).

number of pixels available on high-resolution wall-sized displays $(20,480 \times 6,400$ for WILD) and the possibility of viewer locomotion, it is not easy at first to know how to exploit these capabilities.

To illustrate this, suppose we need to show a map on a wall-sized display for demonstration purposes. A naive approach takes an existing map and enlarges it to the entire wall. Although from far the map could appear more impressive than on a desktop screen (a "movie theater" effect), viewers have no incentive to come closer because no additional detail is shown. This is illustrated by the red curves on Fig. 7a. These curves reproduce three of the functions from Fig. 5 but correct them for the amount of features present in a stretched map image. ${ }^{6}$ We can see that no extra information can be gathered when getting closer than $2 \mathrm{~m}$. An alternative approach is to stitch together multiple full-resolution map images to create a ultra-high-resolution map that occupies the entire wall. This approach may also produce impressive results, but it does not fully exploit the potential of the large display environment either. This is illustrated by the dashed blue curves in Fig. $7 \mathrm{a}^{7}$ : Features of $16 \mathrm{ppc}$ (rivers and parks) are clearly visible from far, but features of $4 \mathrm{ppc}$ (street names) are illegible.

\subsubsection{A Better Visualization}

Re-designing maps from scratch for multiple viewing distances is hard. The same is true for other visualizations. Hybrid-image visualizations can be used as a generic solution to this problem, as they make it possible to combine two visualizations designed for different viewing distances into a single image. Hybrid-image visualizations employ the same basic technique as the original hybrid images [30, 34]:

1. An image we refer to as the near image is high-pass filtered.

2. A different image, the far image, is low-pass filtered.

3. After filtering, the two images are alpha-blended.

In contrast with Oliva et al. [30, 34] who use frequency-domain filters, we perform high-pass and low-pass filtering using Gaussian convolution. We define the radius $r$ of the high-pass and low-pass filters as three times their standard deviation: $r=3 \sigma$.

Fig. $7 \mathrm{~b}$ shows the spatial frequencies of the same map images before alpha-blending: the red curve shows the subway map after applying a 10-pixel high-pass filter while the dashed blue curve shows the full-resolution map after applying a 15-pixel low-pass filter. The key features of the subway map such as text (about $30 \mathrm{ppc}$ ) are visible from far, the details of the full-resolution map are visible from close, and the two images don't overlap much in spatial frequencies. If alternatively the two images from Fig. 7a were blended, the large overlap would

${ }^{6}$ We performed a spatial frequency analysis of a $1024 \times 855$ subway map stretched to a resolution of $7680 \times 6400$.

${ }^{7} \mathrm{We}$ assembled detailed map views of the same city $(1.6 \mathrm{~m} /$ pixel) into a $7680 \times 6400$ image and performed the same analysis. 
make them mostly illegible from both near and far viewing distances. Note, that in hybrid images there is no single distance at which the two percepts suddenly switch. As shown in Fig. 4, visual features do not abruptly transition from being visible to being invisible. Rather, the transition is progressive and there are a range of distances at which both images are visible, although one image typically stands out while the other one appears faint or washed out.

In addition to the basic hybrid-image technique, our implementation of hybrid-image visualizations provides the option to add an unprocessed background image layer, and has a few additional settings:

1. Background image layer: (no settings)

2. Near image layer:

- Radius: the radius of the high-pass filter.

- Transparent: if activated, this option makes the near image transparent in the regions where most frequencies have been filtered out by the high-pass (i.e., $50 \%$ gray pixels).

- Contrast \& brightness: since high-pass filtering often washes out images (see Fig. 7b), these parameters allow to enhance the image contrast after filtering.

- Opacity: opacity used to blend the near image with the background image.

3. Far image layer:

- Radius: the radius of the low-pass filter.

- Opacity: opacity used to blend the near image with the background image.

4. Global settings:

- Contrast \& brightness: optional final contrast enhancement.

Note that, in principle, hybrid images can combine more than two images using band-pass filters, but these may then require very large viewing distances and display sizes. For Fig. 7b, for example, the main features of the far image (about $30 \mathrm{ppc}$ ) only disappear at a viewing distance of $25-30 \mathrm{~m}$, beyond which a third image could be displayed.

\section{Hybrid-IMAge Visualizations}

When creating visualizations for large viewing environments, designers can use hybrid-image techniques to produce a wide range of different effects. Near and far images can show different versions of the same visualization, use different visual representations, or even entirely different datasets. Moreover, there are a multitude of different ways to generate and combine the near and far images depending on intended tasks. In this section we first describe four example hybridimage visualizations that illustrate a range of different applications of the approach. All of these visualizations use hybrid images, but each employs a different combination of techniques to address a specific usage scenario. In the second half of this section we give an overview of some of the distant-dependent data encoding techniques we found most useful in our designs and discuss general recommendations.

\subsection{Example Designs}

Next we demonstrate a range of possible applications of hybrid-image visualizations. Our examples have been tailored to the viewing distances and display configuration of the WILD display, however, designers can use the tools we describe in Section 6 to extend and adapt them to other environments. Full-sized versions of all of the visualizations can be viewed at http://aviz.fr/hybridvis/.

\subsubsection{Dual-Scale Scatterplot}

Large viewing environments can make it possible to display and explore considerable amounts of quantitative data at once. In Fig. 8 we use a hybrid visualization to plot a Hertzsprung-Russell diagram comparing the color index (x) and absolute magnitude (y) of the 30,000 stars closest to Earth. From close to the display, it is possible to examine the dataset at the level of individual stars. However axes and point sizes that are appropriate for close viewing are difficult to see from further away and gaining an overview of the data becomes difficult.

We add a far view in which the properties of the scatterplot are changed to help viewers understand the global structure of the dataset.
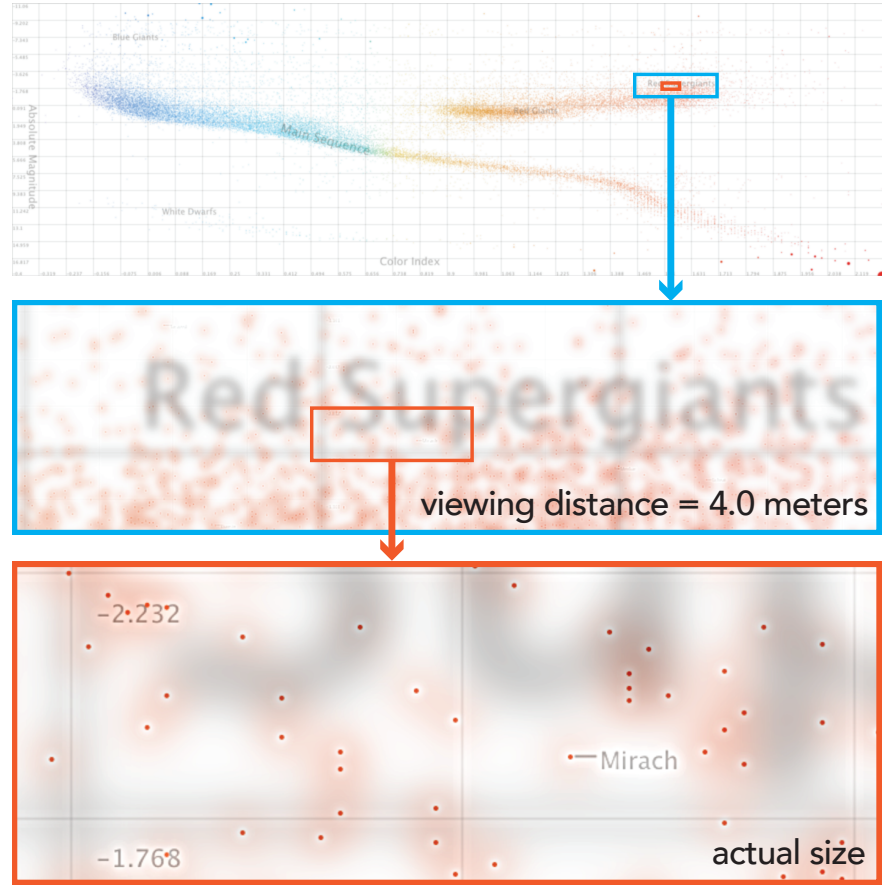

Fig. 8. A dual-scale scatterplot comparing the magnitude and color index of 30,000 stars. Fine points, grids, and labels are visible from close to the display, while larger-scale points and grids support perceptual grouping from a distance.

In the far image, we render stars using larger points that can be visually aggregated when viewed from several meters back (reinforcing perceptual grouping). Fig. 8-bottom shows the combined near and far images as seen from close to the display. The far image's larger points and labels are clearly visible from a $4 \mathrm{~m}$ distance, but recede as the viewer approaches the wall and do not distract from reading the points, grid lines, and star labels in the near image.

When plotting this many data points on a large surface, important values like outliers can be easily missed. To address this, we also use the far image to highlight outliers by scaling the size of each point in the far image based on the distance to its nearest on-screen neighbor. The top image in Fig. 8 includes several such points towards the bottom right. This scaling makes it possible to search for and examine isolated stars that might have been difficult to discover otherwise.

To support viewing at both distances, we also add an aligned dualscale grid, that allows both near and far viewers to read axis values and use a grid to compare points. In addition to finer, more closely-spaced gridlines, the near image contains axis labels at the edge of each screen in the display, allowing close viewers to read values without having to look all the way to the edge of the wall display.

\subsubsection{Dual-Scale Network Diagram}

The dual-scale network visualization in Fig. 9 shows the co-authorship network of our research center (INRIA Saclay). When close to the display, the visualization shows the full network, including about 3,400 authors, 47 research groups, and 21,300 links. Each author in the network is connected to another if they co-authored at least one publication together. Viewed from afar such a network graph becomes quickly unreadable as it consists mostly of small nodes that represent authors and thin links representing the connections between them.

We experimented with different approaches to enhance the visualization for far viewing. Initially, we drew larger versions of all nodes and edges to support visual aggregation (similar to our approach for the hybrid scatterplot). However, drawing thicker lines led to edge congestion and made it difficult to discriminate clusters. Instead, we opted to show only the meta-structure of the network in the far view, plotting only the names of research groups and the connections between them. This layer is optimized to be seen from afar and is designed so that it will not visually interfere with the dense graph. The far visualization 


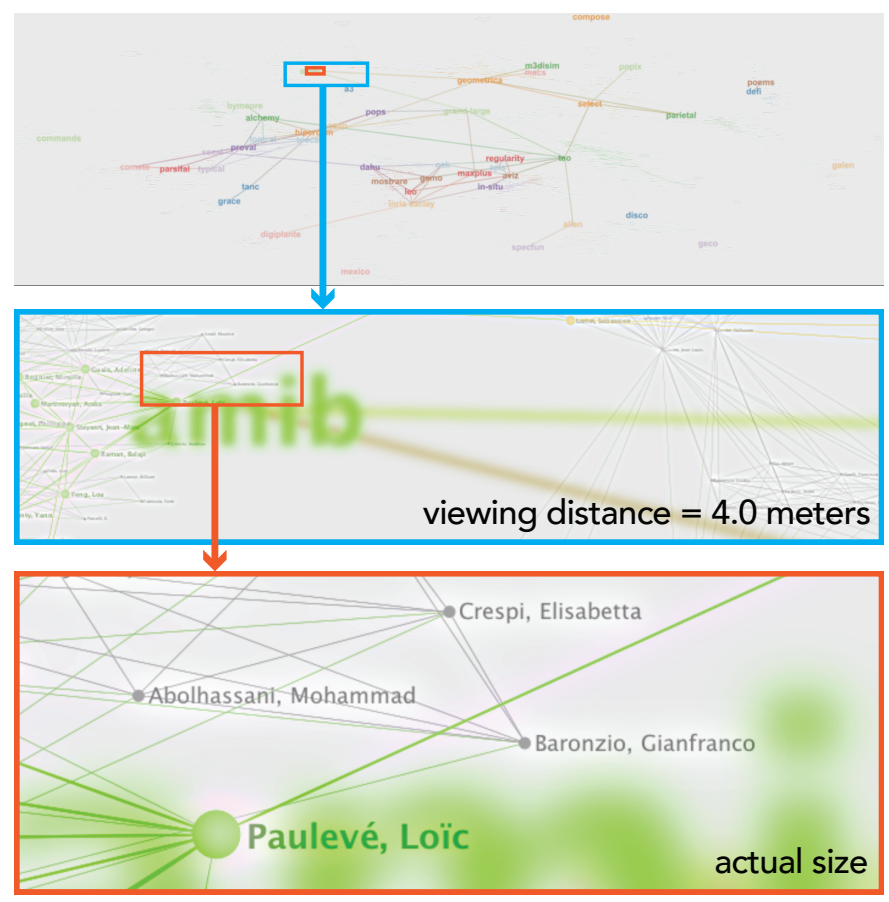

Fig. 9. A dual-scale network diagram showing the co-authorship network within an organization. From a distance, viewers see the relationships between groups, while from close by, they can see the relationships between individual authors.

is visually aligned with the complete co-authorship network, allowing viewers to transition smoothly between the two views.

\subsubsection{Wall-Scale TreeMap}

In theory, the layout of hierarchical structures in treemaps can automatically generate a representation that is amenable to distance-dependent viewing. Rendering appropriate frames around groups of nodes, can further enhance the reading of the structure [41]. Unfortunately, hierarchically nested visualizations like TreeMaps often suffer from occlusion problems, where the labels and borders of higher-level nodes can hide details deeper in the tree. One way to solve this problem is to add additional space around each node and render labels into the frame. While this would make the overall hierarchical structure more visible from afar, labels would be practically invisible. Our wall-scale TreeMap uses a hybrid-image rendering to address these labeling and grouping problems without the use of framing space. Our design supports progressive reveal, where high-level labels and node borders are salient from a distance but become less visible close to the display.

The TreeMap in Fig. 10 shows scientific classifications of living organisms (classifA data from the infovis benchmark repository[13]). With 15 levels and 190,265 nodes the tree is very large. We rendered the borders for nodes in the first four levels and labels for the first two levels into the far image so they could be seen from a distance. Meanwhile, the borders and labels (where possible) for all deeper nodes were rendered in the near image. Because color is used to communicate the depth of leaves at all levels, we rendered filled nodes into the separate background image layer to avoid distortion due to blending and frequency filtering. As Fig. 10-middle shows, the borders and labels of higher level nodes overlap deeper nodes but, due to the blur and blending, this overlay does not hinder the readability of the leaves.

\subsubsection{Small-Multiple Bars with Time Series}

The near and far images can also contain very different representations of the data. In Fig. 11 we demonstrate mixing multiple visualizations by overlaying two different renderings of historical temperature data from 32 European cities. From a distance, viewers see small multiple bar charts showing the change in the average temperature between 1990 and 2012 for each month of the year. However, from closer to the display, the bar charts become less salient and are replaced by small
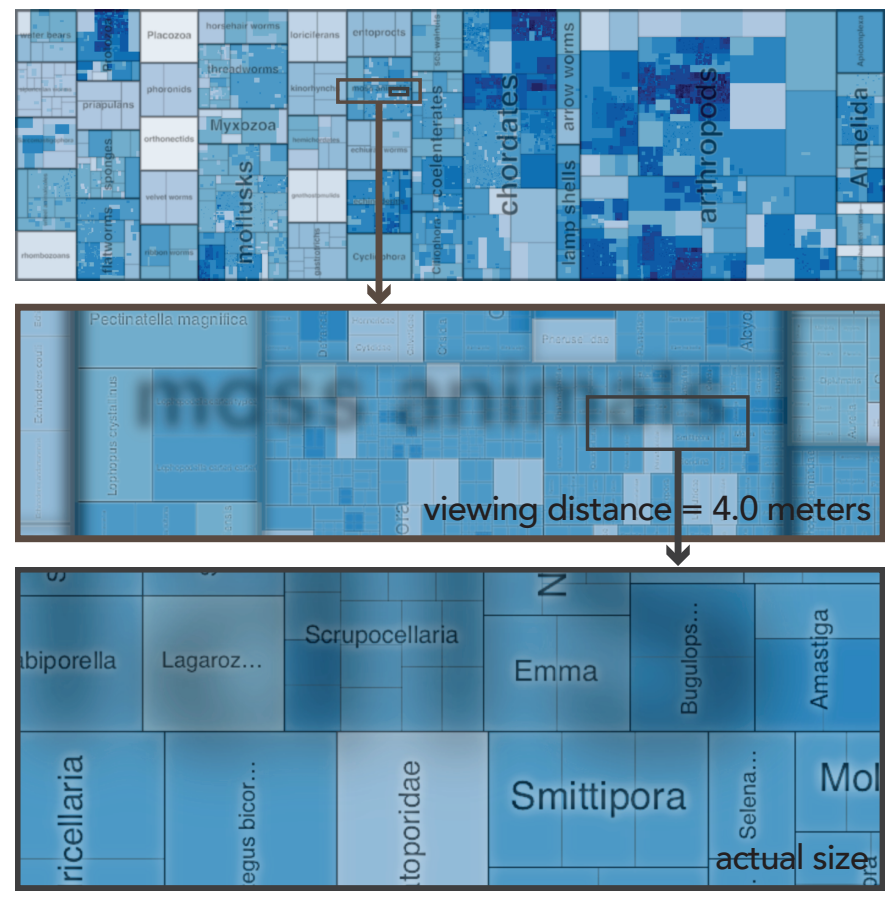

Fig. 10. In a wall-scale treemap, high-level edges and labels are rendered so that they are visible from a distance but become less visible close to the display. These larger marks support navigation from afar, but do not occlude finer details.

time series showing the individual daily temperature readings in that city for every day and year. Viewers can examine the bar charts from a distance to identify larger temperature trends and identify cities which are outliers, then move closer to inspect and compare the raw data. Using a representation with mostly low spatial frequencies (bar charts) for the far image and one with mostly high frequencies (small line charts) for the near image minimizes the interference between the two.

\subsection{Techniques for Hybrid-Image Encoding}

When generating hybrid-image visualizations, designers need to ensure that the information carrying components in each image are not removed by frequency-based filtering or distorted by blending the two images. However, the quality of hybrid-image visualizations depends not only on the filtering parameters used to combine the two images, but also on the properties of the visual marks that make up each image and on the tasks they are designed to support. As a result, it is difficult to give universal recommendations on how to combine visual representations. Nevertheless, from our examples, we have identified a number of techniques that employ hybrid images to support common tasks:

Supporting Perceptual Grouping: One of the chief benefits of large displays is the ability to plot large numbers of data points at once. For viewers who are close to the display, small marks are often desirable because they increase the amount of information that can be explored. However, for viewers further from the display, larger marks are easier to see and may support perceptual aggregation. Rendering the same marks at different sizes for near and far viewing can support both tasks, and allows users to transition between them fluidly.

Highlighting Important Values: When rendering datasets using small marks on a large display, outliers may be difficult to notice. For tasks like visual search where locating outliers may be important, they can be accentuated in the far image.

Reference structures: Grids, axes, and labels are important for interpreting many standard visualizations such as scatterplots and bar charts. However, labels and grids that are readable far from the display may be too large when viewed from close by. Overlaying aligned grids and axis labels at two scales provides both near and far viewers with reference marks at a scale appropriate to their viewing distance. In our experience, dual-scale grids can often be rendered such that both the near and far gridlines effectively disappear when viewed from the 


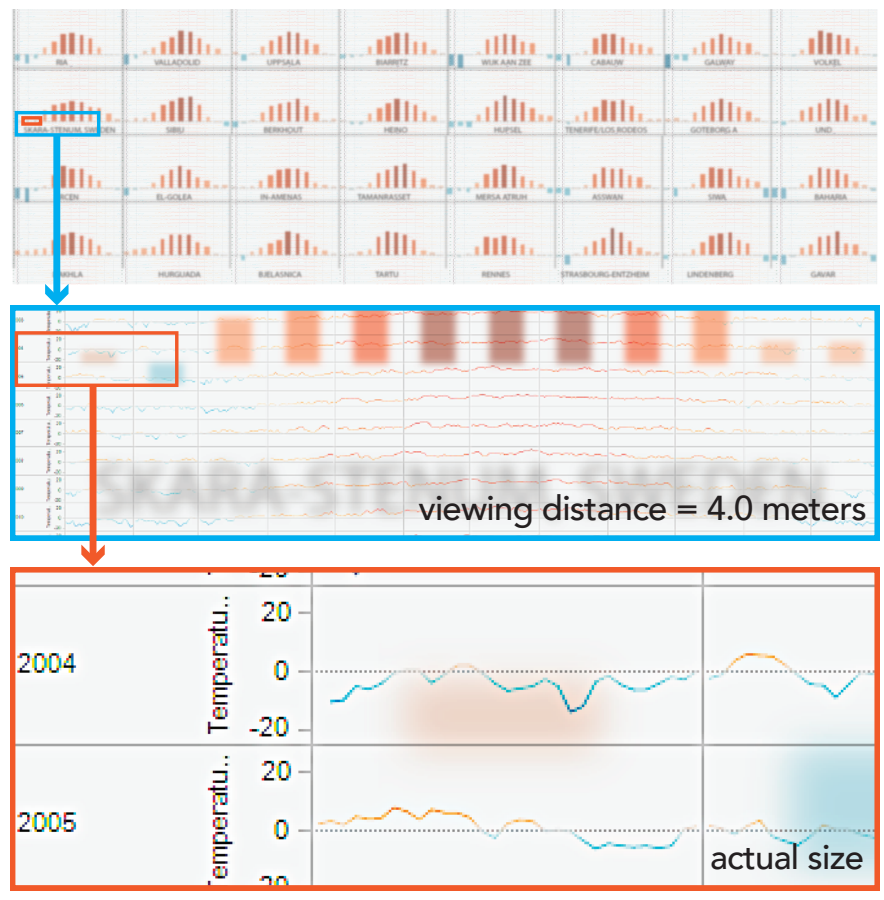

Fig. 11. A hybrid-image visualization containing different representations at each scale. From a distance, viewers see bar charts showing the average temperature shift by month between 1990 and 2012 for 32 major cities in Europe. From close by, they can inspect and compare the individual temperature measurements by day for each of the 22 years.

other distance. The technique of dual-scale grids corresponds to Oliva et al.'s recommendation of aligning edges (see also Fig. 8).

Navigation aids and summaries: The representation for the far viewing distance can be chosen such that it provides entry points to the data, such as regional summaries, data aggregations, or highlights. Adding overlays with summary information on top of existing visualizations can also provide information that would be difficult to mentally integrate (e.g. accurate mean values).

Unobtrusive Labeling Sometimes labels and other textual annotations can interfere with important visual variables. For example, labels on a scatterplot can give a misleading impression of high data density, since they may be much bigger than the points they annotate. In a hybrid-image visualization, details like labels can be effectively hidden by placing them in the near image. This prevents them from interfering with other visual variables when viewed from afar, but still permits them to be easily accessed by approaching the display.

Progressive Reveal: Visualizations such as TreeMaps that are already multi-scale lend themselves well to large-viewing environments. However, features like the labels and boundaries of high-level nodes, which are necessary for navigation from a distance, may actually occlude smaller features that are relevant when close to the display. Splitting the data to show low-resolution data [26] in the far image and highresolution in the near image can mitigate occlusion problems.

Mixing Multiple Visualizations: In some cases, overview and detail views of a dataset may call for different representations to be blended. Frequencies and colors present in both representations need to have minimal overlap so that both can be perceived effectively. Visual mappings that contain mostly fine-grained details (thin lines, small points, and glyphs) and use less color are typically more appropriate for the near image, while those with large features make for better far images.

\section{Tools for Authoring Hybrid-Image Visualizations}

As illustrated by the examples above, designing visualizations that work well at multiple scales requires considerable care. In addition to controlling the frequency content of the near and far images, designers must also tailor the color, type, size, marks, and other aspects of the visualization to ensure that it serves the intended task. This work requires strong intuition about what information will and will not be visible at various distances. However, building that intuition can be

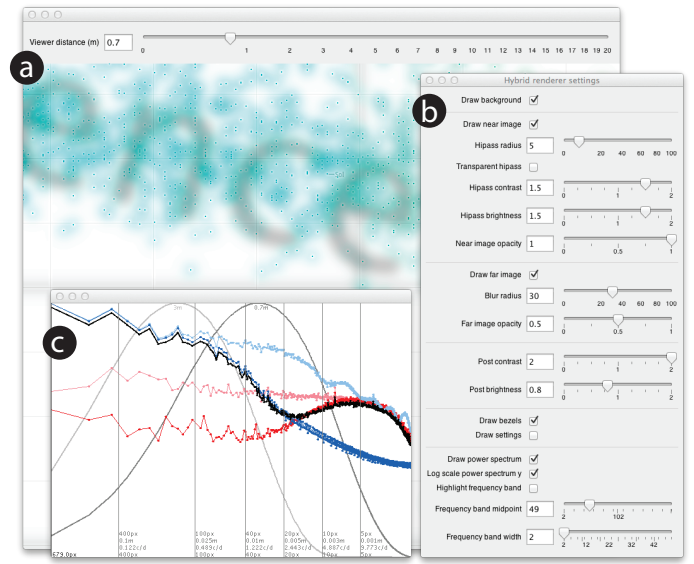

Fig. 12. Interactive preview tools for reviewing and refining hybrid-image visualizations. Designers can preview the image as seen from different distances (a) and can use the control panel (b) and power spectrum visualizer (c) to refine their visualizations and tune how images are processed and blended composited.

difficult, especially because design and development often take place on small displays rather than in large viewing environments.

As a result, visualization designers must carefully balance the tradeoffs between different representations and may need to iteratively tune the parameters of the visualizations and the compositing process. To address this need, we have built a set of tools to support the construction of hybrid-image visualizations. These include (1) a hybrid-image visualization rendering framework, (2) a set of interactive profiling tools for tuning visualizations, and (3) libraries for dynamically sizing common visualization elements like labels text and gridlines.

\subsection{Hybrid-Image Rendering Framework}

We provide a Java-based framework that handles the bulk of the work required to create hybrid visualizations and that we plan to release as an open-source package. It is compatible with a range of existing Javabased visualization toolkits. To generate a visualization, designers extend the base HybridImageRenderer class and implement two drawing methods-one to render the near visualization (seen from close to the display) and one to render the far visualization (seen from further away). When rendering the visualization, the framework performs a low-pass filter on the far image to remove high-frequency information and a high-pass filter on the near image to remove low-frequency information. The framework then composites the two layers together and adjusts their contrast to produce a final visualization.

The tool provides an interactive preview of the final image (Fig. 12a), along with a slider for simulating a range of viewing distances. Designers working on smaller displays can dynamically adjust the simulated viewing distance to understand how the visualization will appear on a large-scale display. Designers can also use an interactive control panel (Fig. 12b) to dynamically tune the parameters of the high- and low-pass filters and adjust the contrast of the composited image. Once the final parameters have been chosen, designers can export the visualization for viewing on a large-scale display.

\subsection{Interactive Profiling}

To help designers tune hybrid-image parameters and make adjustments to their visualizations, we also provide interactive profiling tools. A power spectrum visualizer (Fig. 12c) shows the range of frequencies present in the near and far images, as well as in the final image.

To generate this view, we examine the frequency content of the near and far images both before and after they are filtered. In each case, we perform a Fast-Fourier Transform to convert the image from the spatial domain into the frequency domain. We then radially bin the frequencies to produce a 1-D distribution of the frequencies present in the image, regardless of orientation. The power spectrum visualizer displays the log-scaled distribution of frequencies in each image, with low frequencies at the left of the chart and high frequencies at the right. 


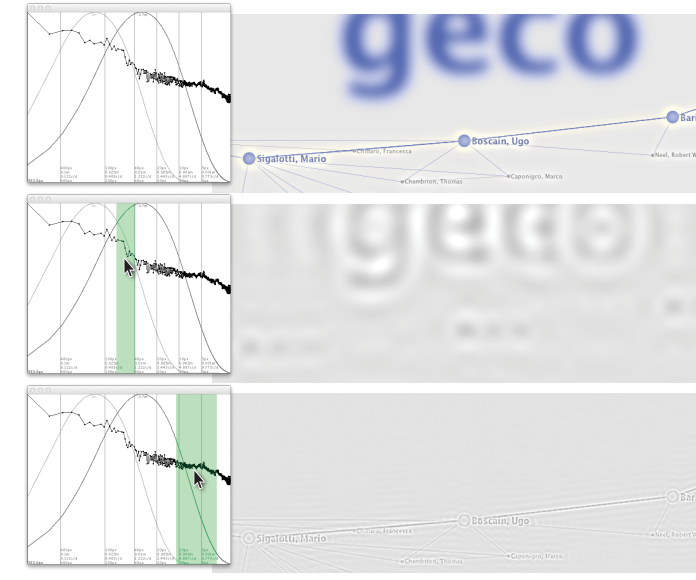

Fig. 13. Clicking on the power spectrum visualizer highlights corresponding frequencies in the visualization preview. Designers can use this to inspect a hybrid-image visualization (top) and identify components that contribute high-frequency (middle) or low-frequency detail (bottom).

The power spectrum of the far image is shown in light blue (before lowpass filtering) and dark blue (after), while the near image is shown in light red (before high-pass filtering) and dark red (after). The power spectrum of the final, composited image is shown in black. These allow designers to better understand the impact of high and low-pass filtering on the frequency content of the image.

Power spectra are also overlaid with plots showing the theoretical contrast-sensitivity response of the human eye at various viewing distances (as described in Section 4.3.1). Using these curves as a reference, designers can interactively refine the high- and low-pass filters, as well as the parameters of their visualizations, in order to make them more or less visible from a given distance.

Often, designers need to adjust aspects of the visualizations themselves-changing the weights of lines and glyphs or altering patterns in order to tailor them to a particular viewing distance. However, determining which components of a visualization contain high- and low-frequency detail is often unintuitive. For example, blocks of text may contain many different frequencies, corresponding to the sizes of individual strokes, words, and even paragraphs rather than just the size of individual letters. This can make it difficult for a designer to determine which aspects of a visualization should be changed in order to alter its frequency distribution. To help designers identify the parts of the image that contain a particular frequency, we also provide a frequency-highlight mode (Fig. 13). In this mode, designers can interactively click on the power spectrum visualizer to highlight a range of frequencies. The system then applies a band-pass filter to the image, removing information from all frequencies outside of the selected range. A designer can use the resulting image to quickly identify which pieces of the visualization might need to be adjusted.

\subsection{Libraries for Common Visualization Components}

A fully automated hybrid-image visualization method is impossible because CSFs and other perceptual models only capture the visibility of spatial frequencies, not the legibility of the features that are important for a given task. We can, however provide tools that predict the legibility of well-known and stable features like text and grids and adjust them with minimal designer input.

\subsubsection{Text Legibility}

Through informal experimentation while building hybrid-image visualizations, we discovered that the ratio between the blur radius and letter width (more specifically the average advance of all lower-case letters of the font) was an excellent predictor of text legibility. $\mathrm{Al}-$ though lower-case letter height is occasionally used as a measure of font legibility [33], we found letter width to be a much better predictor across different typefaces.

More specifically, a ratio between blur radius and letter width above 0.25 produces illegible text. A ratio between 0.10 to 0.15 produces text that can be comfortably read. A ratio below 0.10 increases sharpness but does not yield much difference. Based on this, we implemented a library that allows designers to compute the optimum font size for a given font, blur radius and target legibility (typically between 0.10 or 0.15). It also allows designers to compute the optimum blur radius for a given font, font size and target legibility, and can dynamically derive hybrid-image settings for visualizations like word clouds where the minimum font size is not known in advance.

\subsubsection{Dual-Scale Grids}

Even more so than text, reference structures like grids contain predictable ranges of frequencies and tend to support the same basic tasks. Good settings for the grid alpha and contrast to produce usable and unobtrusive grids are also well-established [5]. Thus, when viewing distances and the parameters of the high- and low-pass filters are known, we can use a CSF to dynamically adjust the size and weight of the grid and ensure that appropriate grids will be visible from each distance. Based on this observation, we provide a library for generating aligned dual-scale grids and labels. Designers specify grid boundaries, the desired density of gridlines, and formatting of the axes. The library then handles grid rendering for both the near and far images.

\section{CONCLUSION}

While the phenomenon of hybrid images has been known for quite some time, this work presents new practical applications of the technique for information visualization. In large-viewing environments where physical locomotion is common, hybrid-image visualizations represent a solution for providing viewing distance dependent overview+detail views in a single image. As such, they support collaboration on a single visualization and leverage implicit, physical interaction through locomotion to change views, without requiring technical equipment such as motion capturing systems.

That said, there are also some limitations to the technique. Depending on the chosen image size as well as filter implementation and filter settings, one view can take considerable time to compute. For our 131 million pixel display, each visualization can take up to $10 \mathrm{~min}$ utes to compute using a current desktop or laptop computer. To support more rapid design cycles, we provide tools that simulate viewing distances and let designers tune parameters quickly. Employing hardware acceleration should make it easier to render such images in realtime, but for now slow render times make these images best-suited for static viewing scenarios. In addition, as with many other visualization techniques, there is no one-size-fits all solution for creating effective hybrid-image visualizations. That means, in domains where data representations need to change quickly based on incoming data or due to changing data characteristics, the use of hybrid-image visualizations would likely be less appropriate and other detail-and-context techniques should be examined.

Our work provides a perceptual background for using hybridimages to visualize data and provides a detailed rationale to aid others in designing them. Our set of example visualizations also highlights the technique's wide range of applications. Many of the techniques we describe can be used to enhance existing wall-size display visualization without interfering with existing context, for example by adding reference structures for far viewing. More generally, the techniques are also applicable to non-digital, non-collaborative, and non-work environments. For example, hybrid-image visualizations can be used on posters or billboards in public places where the progressive reveal of content could entice passers-by to examine the display more closely and spend more time with it than they would have otherwise. We hope our work has laid the foundation for a range of future applications.

\section{ACKNOWLEDGMENTS}

This work is sponsored by the French Research Organization, project grant ANR-11-JS02-003.

\section{References}

[1] S. Afzal, R. Maciejewski, Y. Jang, N. Elmqvist, and D. Ebert. Spatial Text Visualization Using Automatic Typographic Maps. Transactions on 
Visualization and Computer Graphics, 18(12):2556-2564, 2012. doi> 10. 1109/TVCG.2012.264

[2] C. Andrews, A. Endert, B. Yost, and C. North. Information Visualization on Large, High-resolution Displays: Issues, Challenges, and Opportunities. Information Visualization, 10(4):341-355, 2011. doi> 10.1177/ 1473871611415997

[3] T. Ballendat, N. Marquardt, and S. Greenberg. Proxemic Interaction: Designing for a Proximity and Orientation-Aware Environment. In Proc. Interactive Tabletops and Surfaces (ITS), pp. 121-130. ACM, 2010. doi> $10.1145 / 1936652.1936676$

[4] P. Barten. Contrast Sensitivity of the Human Eye and its Effects on Image Quality. SPIE Press Series. SPIE Optical Engineering Press, 1999.

[5] L. Bartram and M. Stone. Whisper, Don't Scream: Grids and Transparency. IEEE Transactions on Visualization and Computer Graphics, 17(10):1444-1458, 2011. doi> 10.1109/TVCG.2010.237

[6] S. Battiato, G. Di Blasi, G. M. Farinella, and G. Gallo. Digital Mosaic Frameworks - An Overview. Computer Graphics Forum, 26(4):794-812, 2007. doi> 10.1111/j.1467-8659.2007.01021.x

[7] P. Baudisch and C. Gutwin. Multiblending: Displaying Overlapping Windows Simultaneously without the Drawbacks of Alpha Blending. In Proc. Human Factors in Computing Systems (CHI), pp. 367-374. ACM, 2004. doi> 10.1145/985692.985739

[8] M. Beaudouin-Lafon, O. Chapuis, J. Eagan, T. Gjerlufsen, S. Huot, C. Klokmose, W. Mackay, M. Nancel, E. Pietriga, C. Pillias, R. Primet, and J. Wagner. Multi-surface Interaction in the WILD Room. Computer, 45(4):48-56, 2012. doi> 10.1109/MC.2012.110

[9] B. B. Bederson and J. D. Hollan. Pad++: A Zooming Graphical Interface for Exploring Alternate Interface Physics. In Proc. User Interface Software and Technology (UIST), pp. 17-26. ACM, 1994. doi> 10.1145/ 192426.192435

[10] A. Bezerianos and P. Isenberg. Perception of Visual Variables on Tiled Wall-Sized Displays for Information Visualization Applications. IEEE Transactions on Visualization and Computer Graphics, 18(12):25162525, 2012. doi> 10.1109/TVCG.2012.251

[11] R. N. Bracewell. Fourier Analysis and Imaging. Springer, 2003.

[12] F. W. Campbell and J. G. Robson. Application of Fourier Analysis to the Visibility of Gratings. The Journal of Physiology, 197(3):551-566, 1968.

[13] Catherine Plaisant and Jean-Daniel Fekete. Information Visualization Repository. Online article, http://www.cs.umd.edu/hcil/ InfovisRepository/, visited: 2013.

[14] A. Dillon. Reading from Paper versus Screens: A Critical Review of the Empirical Literature. Ergonomics, 35(10):1297-1326, 1992. doi> 10. 1080/00140139208967394

[15] D. M. Eagleman. Visual Illusions and Neurobiology. Nature Reviews Neuroscience, 2(12):920-926, 2001. doi> 10.1038/35104092

[16] A. Endert, C. Andrews, Y. H. Lee, and C. North. Visual Encodings that Support Physical Navigation on Large Displays. In Proc. Graphics Interface (GI), pp. 103-110. CHCCS, 2011.

[17] M. S. Farid and A. Mahmood. Image Morphing in Frequency Domain. Journal of Mathematical Imaging and Vision, 42(1):50-63, 2012. doi> 10.1007/s10851-011-0273-3

[18] G. W. Furnas. A Fisheye Follow-up: Further Reflections on Focus + Context. In Proc. Human Factors in Computing Systems (CHI), pp. 9991008. ACM, 2006. doi> 10.1145/1124772.1124921

[19] E. B. Goldstein. Sensation and Perception. Brooks/Cole Publishing, Pacific Grove, USA, $5^{\text {th }}$ edition, 1999.

[20] S. Greenberg, N. Marquardt, T. Ballendat, R. Diaz-Marino, and M. Wang. Proxemic Interactions: The new Ubicomp? Interactions, 18(1):42-50, 2011. doi> 10.1145/1897239.1897250

[21] C. Harrison and S. E. Hudson. A New Angle on Cheap LCDs: Making Positive Use of Optical Distortion. In Proc. User Interface Software and Technology (UIST), pp. 537-540. ACM, 2011. doi> 10.1145/2047196. 2047266

[22] N. Henry, J.-D. Fekete, and M. J. McGuffin. NodeTrix: A Hybrid Visualization of Social Networks. Transactions on Visualization and Computer Graphics, 13(6):1302-1309, 2007. doi> 10.1109/TVCG.2007.70582

[23] E. W. Ishak and S. K. Feiner. Interacting with Hidden Content using Content-aware Free-space Transparency. In Proc. User Interface Software and Technology (UIST), pp. 189-192. ACM, 2004. doi> 10.1145/ 1029632.1029666

[24] D. Keim, M. Hao, U. Dayal, M. Hsu, and J. Ladisch. Pixel Bar Charts: A New Technique for Visualizing Large Multi-Attribute Data Sets without Aggregation. In Proc. Symposium on Information Visualization (InfoVis), volume 0, pp. 113-120. IEEE, 2001. doi> 10.1109/INFVIS.2001.963288 [25] S. Kim, X. Cao, H. Zhang, and D. Tan. Enabling Concurrent Dual Views on Common LCD Screens. In Proc. Human Factors in Computing Systems (CHI), pp. 2175-2184. ACM, 2012. doi> 10.1145/2207676. 2208369

[26] H. Lam, T. Munzner, and R. Kincaid. Overview Use in Multiple Visual Information Resolution Interfaces. IEEE Transactions on Visualization and Computer Graphics, 13(6):1278-1285, 2007. doi> 10.1109/TVCG. 2007.70583

[27] H. Lieberman. Powers of Ten Thousand: Navigating in Large Information Spaces. In Proceedings of the Symposium on User Interface Software and Technology (UIST), pp. 15-16. ACM, New York, NY, USA, 1994. doi> 10.1145/192426.192434

[28] M. Nacenta, U. Hinrichs, and S. Carpendale. FatFonts: Combining the Symbolic and Visual Aspects of Numbers. In Proc. Advanced Visual Interfaces (AVI), pp. 407-414. ACM, 2012. doi> 10.1145/2254556. 2254636

[29] A. Oliva and P. G. Schyns. Coarse Blobs or Fine Edges? Evidence that Information Diagnosticity Changes the Perception of Complex Visual Stimuli. Cognitive Psychology, 34(1):72-107, 1997. doi> 10.1006/cogp.1997. 0667

[30] A. Oliva, A. Torralba, and P. G. Schyns. Hybrid Images. ACM Transactions on Graphics, 25(3):527-532, 2006. doi> 10.1145/1141911. 1141919

[31] V. Ostromoukhov and R. D. Hersch. Artistic Screening. In Proc. Computer Graphics and Interactive Techniques (SIGGRAPH), pp. 219-228. ACM, 1995. doi> 10.1145/218380.218445

[32] H. Pedersen and K. Singh. Organic Labyrinths and Mazes. In Proc. NonPhotorealistic Animation and Rendering (NPAR), pp. 79-86. ACM, 2006. doi> 10.1145/1124728.1124742

[33] V. Rossum. Measuring Font Legibility. Unpublished manuscript, http://homepages.inf .ed.ac.uk/mvanross/reprints/ legibility.pdf, 1998. visited: 2012.

[34] P. G. Schyns and A. Oliva. From Blobs to Boundary Edges: Evidence for Time and Spatial Scale Dependent Scene Recognition. Psychological Science, 5:195-200, 1994. doi> j.1467-9280.1994.tb00500.x

[35] P. G. Schyns and A. Oliva. Dr. Angry and Mr. Smile: When Categorization Flexibly Modifies the Perception of Faces in Rapid Visual Presentations. Cognition, 69(3):243-265, 1999. doi> 10.1016/S0010-0277(98) 00069-9

[36] M. Stone. In Color Perception, Size Matters. IEEE Computer Graphics and Applications, 32(2):8-13, 2012. doi> 10.1109/MCG.2012.37

[37] K. Swaminathan and S. Sato. Interaction Design for Large Displays. interactions, 4(1):15-24, 1997. doi> 10.1145/242388.242395

[38] USD Internet Psychology Laboratory. The Contrast Sensitivity Function (CSF). Online article, http://sunburst.usd.edu/ schieber/ coglab/CSF Intro.html, visited: 2013.

[39] D. Vogel and R. Balakrishnan. Interactive Public Ambient Displays: Transitioning from Implicit to Explicit, Public to Personal, Interaction with Multiple Users. In Proc. User Interface Software and Technology (UIST), pp. 137-146. ACM, 2004. doi> 10.1145/1029632.1029656

[40] C. Ware. Information Visualization - Perception for Design. Morgan Kaufmann Publishers, Amsterdam, $2^{\text {nd }}$ edition, 2004.

[41] M. Wattenberg and D. Fisher. Analyzing Perceptual Organization in Information Graphics. Information Visualization, 3(2):123-133, 2004. doi> 10.1057/palgrave.ivs.9500070

[42] P. C. Wong, P. Mackey, K. Perrine, J. Eagan, H. Foote, and J. Thomas. Dynamic Visualization of Graphs with Extended Labels. In Proc. Symposium on Information Visualization (InfoVis), pp. 73-80. IEEE, 2005. doi> 10.1109/INFOVIS.2005.11

[43] Y.-J. Yoon, H.-S. Ryu, J.-M. Lee, S.-J. Park, S.-J. Yoo, and S.-M. Choi. An Ambient Display for the Elderly. In Proc. Universal Access in HumanComputer Interaction (UAHCI), pp. 1045-1051. Springer-Verlag, 2007. doi> 10.1007/978-3-540-73281-5_114

[44] B. Yost, Y. Haciahmetoglu, and C. North. Beyond Visual Acuity: The Perceptual Scalability of Information Visualizations for Large Displays. In Proc. Human Factors in Computing Systems (CHI), pp. 101-110. ACM, 2007. doi> 10.1145/1240624.1240639

[45] B. Yost and C. North. The Perceptual Scalability of Visualization. IEEE Transactions on Visualization and Computer Graphics, 12(5):837-844, 2006. doi> 10.1109/TVCG.2006.184 